\title{
Allergic reactions to decorative skin paintings
}

\author{
AF Nikkels, * F Henry, GE Piérard \\ Department of Dermatopathology, University Medical Center of Liège, Liège, Belgium. *Corresponding author, \\ Department of Dermatopathology, CHU du Sart Tilman, B-4000 Liège, Belgium, tel. + 324 3662499; fax +324 3662976; \\ E-mail:af.nikkels@chu.ulg.ac.be
}

\begin{abstract}
Background Dye mixtures containing henna, P-phenylenediamine (PPD), and indigo are applied to the skin in various parts of the world for religious purposes, social recognition or fun.

Aim To identify eventual allergens responsible for contact sensitization to skin decoration in three young children. Lesions remained strictly limited to the site painted during the summer holiday. None of the children had a previous history of contact dermatitis, but two of them suffered from severe seasonal allergic rhinoconjunctivitis.
\end{abstract}

Findings Patch testing revealed severe contact hypersensitivity to PPD in all three patients, and one child presented a positive prick test to $1 \%$ henna in water. The eruptions resolved following spontaneous elimination of the dyes and topical applications of corticosteroids, but left residual depigmentation.

Conclusion PPD is the main allergen identified in allergic reactions to decorative skin paintings.

Key words: allergic contact dermatitis, henna, indigo, lawsone, paraphenylenediamine

\section{Introduction}

Skin painting using henna is part of religious, social and ritualistic traditions in many parts of the world.1,2 Skin decoration is offered to tourists in several countries. Henna is one of the most frequently used dyes. Common red henna (Lawsonia inermis family Lythraceae) is prepared by powdering the dried leaves and stems of Lawsonia, a shrub cultivated in India, North America, North Africa and Sri Lanka. The grey powder is mixed with water to prepare a thick paste that is left on the skin for $5 \mathrm{~min}$ to $24 \mathrm{~h}$. The active staining ingredient is a naphtoquinone (lawsone, 2-hydroxy-1,4-naphthoquinone), responsible for a red-brown dye. P-phenylenediamine (PPD) (dark brown dye) or indigo blue (blue-black dye) are frequently added to darken the lawsone stain. Henna staining is known to be temporary and may last a few weeks. Despite their ubiquitous use, contact sensitization to skin paintings has been reported rarely; ${ }^{2-7}$ PPD allergy has been identified in some cases, $3,4,6,7$ although hypersensitivity to henna has also been reported.2,5,8 We report three children who experienced allergic contact dermatitis to dyes used for skin decoration done during the summer holidays.

\section{Case reports}

\section{Case 1}

On holiday in southern Egypt, a 10-year-old girl received skin decoration on her right arm done with toothpicks drenched in henna dyes. As the picture faded with swimming and washing, the procedure was repeated three to four times. The exact recipe of the dye was unknown. Upon return home she developed an allergic contact dermatitis restricted to the site of the skin decoration (fig. 1). The girl had no previous history of contact dermatitis, but suffered from severe hay fever. Topical corticosteroids were applied and the lesions healed leaving marked hypopigmentation. Patch tests revealed a severe reaction to PPD (Table 1). Strong prick test reactions occurred for Herbacaea (mixtures 1, 2 and 3, Stallergenes, Waterloo, Belgium), weed mixtures (3, 5, 12 grasses, Stallergenes, Waterloo, Belgium) and $1 \%$ henna in water. No reaction to $1 \%$ indigo blue in water was observed. The final diagnosis was allergic contact dermatitis to PPD. 


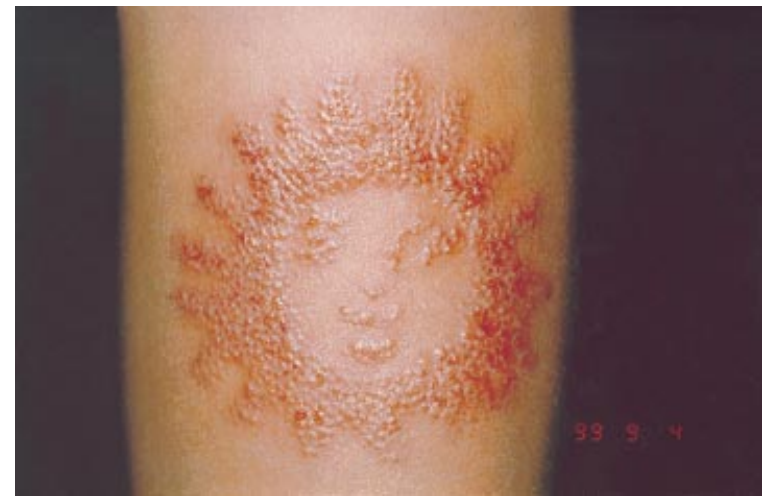

fig. 1 Allergic contact dermatitis to a skin painting on the arm (patient 1).

Table 1 Patch testing results

\begin{tabular}{|c|c|c|c|c|}
\hline \multirow[b]{2}{*}{ Patient } & \multicolumn{2}{|c|}{ European standard battery } & \multirow[b]{2}{*}{$\begin{array}{l}\text { Henna } \\
10 \% \text { W }\end{array}$} & \multirow[b]{2}{*}{$\begin{array}{l}\text { Indigo blue } \\
10 \% \mathrm{~W}\end{array}$} \\
\hline & PPD & $\begin{array}{l}\text { Other } \\
\text { components }\end{array}$ & & \\
\hline 1 & +++ & 0 & 0 & 0 \\
\hline 2 & +++ & 0 & 0 & 0 \\
\hline 3 & +++ & 0 & 0 & 0 \\
\hline
\end{tabular}

W, water.

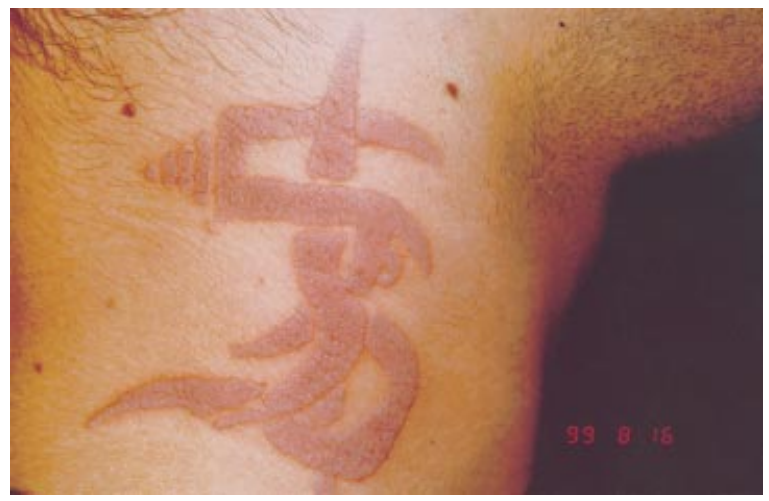

fig. 2 Allergic contact dermatitis to a skin painting on the neck (patient 2)

\section{Case 2}

During the summer holidays in Mallorca, an 17-year-old boy applied skin decorations on his neck using a fine brush. The composition of the dye mixture was unknown. Five days later an erythematous and itchy dermatitis developed at the site of the decoration (fig. 2). The lesions resolved with topical corticosteroids, but with persistent postinflammatory hyperpigmentation. The boy had a previous history of severe hay fever and also had experienced several episodes of phytophotodermatitis. Patch testing revealed severe PPD sensitization (Table 1). The patient also showed strong positive prick test reactions to weed mixtures $(3,5,12$ grasses, Stallergenes,
Waterloo, Belgium), but no reactions were noted to Herbaceae (1, 2 and 3 mixtures, Stallergenes, Waterloo, Belgium), 1\% henna and $1 \%$ indigo in water.

\section{Case 3}

An 8-year-old girl had her arm painted during the summer holidays in Turkey. The drawing was outlined with a pencil and covered with a brown-black paste that remained in place for $24 \mathrm{~h}$. A black skin decoration appeared after the paste was washed off. The percentage of the various ingredients was unknown. As the painting faded during swimming, the procedure was repeated 4 days later. About 8 days later the girl experienced an allergic contact dermatitis restricted to the site of the skin painting. The lesion was treated with topical steroids, but a residual hypopigmentation remained. No personal history of atopy was recorded, although the mother and grandmother suffered from allergic asthma. Patch testing showed a severe positive reaction to PPD (Table 1).

\section{Discussion}

Allergic reactions to henna are rare. ${ }^{2,5,8}$ Indeed, it is often used in hair products as an alternative dye for individuals suffering from contact dermatitis to PPD. However, occupational sensitization may occur in hairdressers and patients using henna-containing hair products or nail varnish. ${ }^{8-13}$ Ectopic contact dermatitis of the eyelids, cheeks and forehead can be related to the use of henna nail varnish. ${ }^{13}$ However, PPD allergy is more frequent in subjects, particularly hairdressers, who frequently use hair products containing PPD derivatives. ${ }^{14}$

The present three cases illustrate allergic contact reactions to occasional skin painting in young children. In all the cases severe PPD contact hypersensitivity was revealed confirming recent reports; $3,4,6,7$ although as the dyes used were not available for skin testing, other ingredients might also have been responsible for allergy. However, the second patient presented a reaction only 5 days after the painting, a relatively rapid onset for contact sensitization. As henna is part of the Herbaceae family and as the patient had a history of hay fever, phytophotodermatitis and a positive prick test to henna, a phytophototoxic reaction cannot be totally excluded as a cofactor.

The results also demonstrate a worrying trend towards the use of PPD in 'henna' dye and tattoos, with subsequent PPD sensitization. As this sensitization occurs in young people, and, as the PPD group of chemicals are the only very effective form of permanent hair dyes, these individuals will be severely disadvantaged when they are older and want to use hair dyes.

\section{References}

1 Pasricha JS, Gupta R, Panjwani S. Contact dermatitis to henna (Lawsonia). Contact Dermatitis 1980; 6: 288-289. 
2 García Ortiz JC, Terron M, Bellido J. Contact allergy to henna. Int Arch Allergy Immunol 1997; 114: 298-299.

3 Wakelin SH, Creamer D, Rycroft RJG, White IR, McFadden JP. Contact dermatitis from paraphenylenediamine used as a skin paint. Contact Dermatitis 1998; 39: 92-93.

4 Gallo R, Ghigliotti G, Cozzani E, Balestrero S. Contact dermatitis from paraphenylenediamine used as a skin paint: a further case. Contact Dermatitis 1999; 40: 57.

5 Lewin PK. Temporary henna tattoo with permanent scarification (letter). Can Med Ass J 1999; 160: 310.

6 Nixon R, Orchard D. Positive para-phenylene diamine (PPD) reactions following paint-on tattoos (letter). Australas J Dermatol 1999; 40: 120.

7 O'Brien TJ, McColl DM. Unusual reactions to paint-on tattoos (letter). Australas J Dermatol 1999; 40: 120.

8 Wantke F, Götz M, Jarisch R. Contact dermatitis due to henna, solvent red 1 and solvent red 3. A case report. Contact Dermatitis 1992; 27: 346-347.

9 Gupta BN, Mathur AK, Agarwal C, Singh A. Contact sensitivity to henna. Contact Dermatitis 1986; 15: 303-304.

10 Natow AJ. Henna. Cutis 1986; 38: 21.

11 Nigam PK, Saxena AK. Allergic contact dermatitis from henna. Contact Dermatitis 1988; 18: 55-56.

12 al-Sheikh OA, Gad el-Rab MO. Allergic contact dermatitis: clinical features and profile of sensitizing allergens in Riyadh, Saudi Arabia. Int J Dermatol 1996; 35: 493-497.

13 Etienne A, Piletta P, HauSeries C, Pasche-Koo F. Ectopic contact dermatitis from henna. Contact Dermatitis 1997; 37: 183.

14 Dawber RPR, Ebling FJC, Wojnarowska T. Disorders of hair. In: Champion RH, Burton JL, Ebling FJG, editors. Textbook of Dermatology 5th edn. Blackwell Scientific Publications, Oxford, 1992: 2533-2538.

\section{Visit the EADV website at: www.eadv.org}

